\title{
Tip of the Iceberg: Roles of CircRNAs in Cancer Glycolysis
}

This article was published in the following Dove Press journal: OncoTargets and Therapy

\author{
Tan $\mathrm{Li}^{\mathrm{I}}$ \\ Hong-chun Xian ${ }^{2}$ \\ Li Dai ${ }^{1}$ \\ Ya-ling Tang ${ }^{2}$ \\ Xin-hua Liang'
}

'State Key Laboratory of Oral Diseases and National Clinical Research Center for Oral Diseases, Department of Oral and Maxillofacial Surgery, West China Hospital of Stomatology, Sichuan University, Chengdu, People's Republic of China; ${ }^{2}$ State Key Laboratory of Oral Diseases and National Clinical Research Center for Oral Diseases, Department of Oral Pathology, West China Hospital of Stomatology, Sichuan University, Chengdu, People's Republic of China

Correspondence: Ya-ling Tang

$\mathrm{Tel}+86 \quad 15008458098$

Fax +86 28-86060II4

Email tangyaling@scu.edu.cn

Xin-hua Liang

Tel $+86 I 5884483965$

Fax +86 28-86060II4

Email Ixh88866@scu.edu.cn

\begin{abstract}
Warburg effect reflects that tumor cells tend to generate energy by aerobic glycolysis rather than oxidative phosphorylation (OXPHOS), thus promoting the development of malignant tumors. As a kind of non-coding RNA, circular RNA (circRNA) is characterized by a closed ring structure and emerges as a regulator of cancer metabolism. Mounting studies revealed that circRNA can regulate the cancer metabolism process through affecting the expression of glycolysis relevant enzymes, transcription factors (TFs), and signaling pathways. In this review, we comprehensively analyzed and concluded the mechanism of circRNA regulating glycolysis, hoping to deepen the cognition of the cancer metabolic regulatory network and to reap huge fruits in targeted cancer treatment.
\end{abstract}

Keywords: cancer, circRNAs, Warburg effect, glucose metabolism

\section{Introduction}

Warburg effect, proposed in the 1920s, remains one of the most obvious metabolic changes in cancer and is a hot research topic. ${ }^{1,2}$ It demonstrates that tumor cells tend to undergo glycolysis even in a normoxic environment (Figure 1), leading to the rapid growth of tumors in many aspects. ${ }^{3,4}$ Warburg effect alleviates oxidative stress damage caused by mitochondria when cancer cells choose glycolysis instead of OXPHOS to generate energy. ${ }^{3,5}$ Besides, glycolysis can produce excess lactic acid that contributes to the avoidance of immune surveillance. ${ }^{6,7}$ Moreover, the intracellular transport of glucose in tumor cells is enhanced, maintaining a highspeed glycolysis process and a high level of ATP/ADP ratio. ${ }^{8-10}$ Last, glycolysis provides a wealth of metabolic intermediates and nutrients, ${ }^{11,12}$ which can be used as raw materials for the synthesis of biological macromolecules (such as fatty acids, nucleic acids, and amino acids). ${ }^{13}$ Previous studies have shown that the Warburg effect may be regulated by oncogenes, tumor suppressor genes, and key enzymes in glucose metabolism, but the underlying mechanisms are incompleted. ${ }^{14-16}$ Pieces of evidence show that circRNA is an emerging regulatory factor of the Warburg effect.

As a special kind of non-coding RNA, circRNA has a covalent continuous closedloop structure, without the 3 'end polyA trimming the 5 'end cap. ${ }^{17}$ The stable structure and conserved sequence of circRNA prevent it from digestion by an exonuclease and make it has a longer half-life compared with linear RNA, thus enabling it as a potential disease biomarker (Figure 2). ${ }^{18}$ As a competitive endogenous RNA (ceRNA), circRNA can act as a micro RNA (miRNA) sponge to competitively bind to miRNA, thereby affecting miRNA-induced gene silencing. Plenty of effort has been put toward recognizing one specific mechanism "miRNA sponging", but only a handful of transcripts 


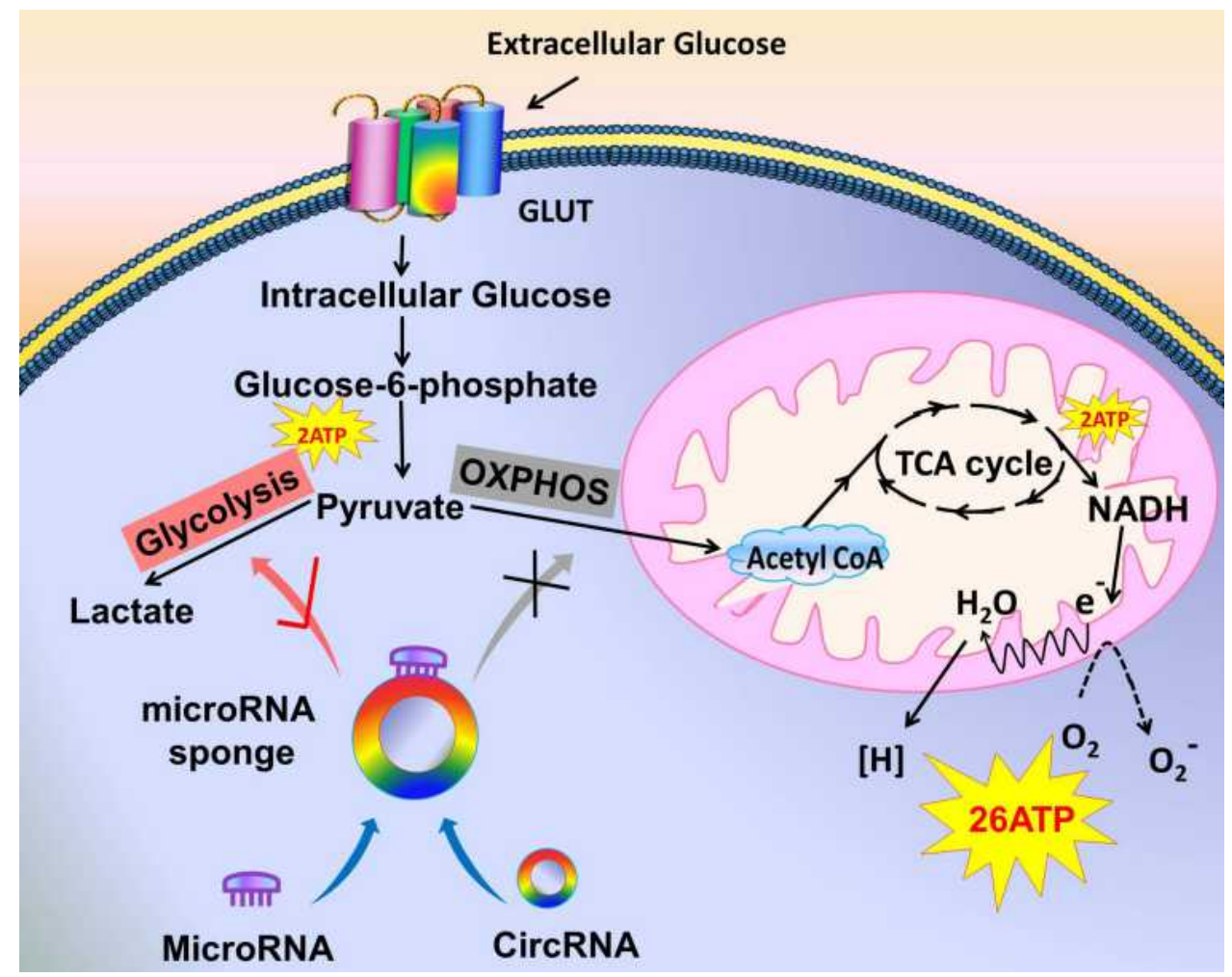

Figure I Tumor cells choose glycolysis over oxidative phosphorylation. The model shows that the extracellular glucose is transported to the cells by GLUT to become intracellular glucose, which is converted to glucose-6-phosphate under the action of enzymes and then convert to pyruvate, producing 2 ATP in this process. On the right, in the normally oxygenated condition, pyruvate is converted to acetyl-CoA by oxidative phosphorylation. Acetyl-CoA enters the TCA cycle to produce 2 ATP and then reproduces 26 ATP via the electron transport chain. On the left, in tumor cells, some circRNAs are abnormally expressed, and they target microRNAs as molecular sponges. Thus, oxidative phosphorylation cannot be carried out normally. Instead, glycolysis is promoted to produce lactate.

really can be considered true "sponges" due to the low number of miRNA response elements. The first reported circRNA, ciRS-7, can regulate the function of miR-7 target gene Fos in human and mouse brains. ${ }^{14}$ Moreover, circRNA can regulate gene transcription, combine with RNA binding protein (RBP) to regulate biological activities of cells, and encode the protein. Besides, mounting latest studies show that circRNA plays a key role in regulating glycolysis in cancer cells. By targeting and binding miRNA, circRNA is involved in the regulation of tumor glucose metabolism and affects the progression of malignant tumors (Figure 3).

In this review, we comprehensively discussed circRNAregulated glycolysis of cancer and elaborated the relevant circRNA-miRNA axes (Table 1), in order to deepen the understanding of tumor aerobic glycolysis regulation network and find new directions for cancer treatment.

\section{CircRNAs in Regulating Transporters of Glycolysis}

Since the metabolism of carbohydrates is a dominating energy supply pathway, a cluster of interrelated enzymes can cooperate to maintain the normal operation of the process smoothly. ${ }^{19-21}$ First of all, the glucose transporter (GLUT) is responsible for the input of glucose. ${ }^{22,23} \mathrm{In}$ order to meet the exorbitant demands of glucose, GLUTs (GLUT1, GLUT2, GLUT3, and GLUT4) all reach a higher level in cancer cells. ${ }^{24-26}$

The fast development of bioinformatics has accelerated the exploration of circRNAs. Studies have shown that certain circRNAs are significantly up-regulated in tumor tissues, affecting cell metabolism and promoting tumor glycolysis, growth, and metastasis. The silence of circHIPK 3 in pancreatic islets can reduce the expression of Slc2a2, which encodes GLUT2. ${ }^{27}$ CircHIPK3 targets miR-124, reduces the expression levels of transporters as well as enzymes, and inhibits glycolysis. ${ }^{28,29}$ Circ-Amotl1 can target AKT1 and PDK1 (pyruvate dehydrogenase kinase 1). ${ }^{30}$ In non-small cell lung cancer (NSCLC), the down-regulated circARHGAP10 can decrease the expression of GLUT1, inhibit the intracellular transport of glucose, glucose consumption as well as lactate formation to inhibit glycolysis through sponging miR-150-5p. ${ }^{31,32}$ 


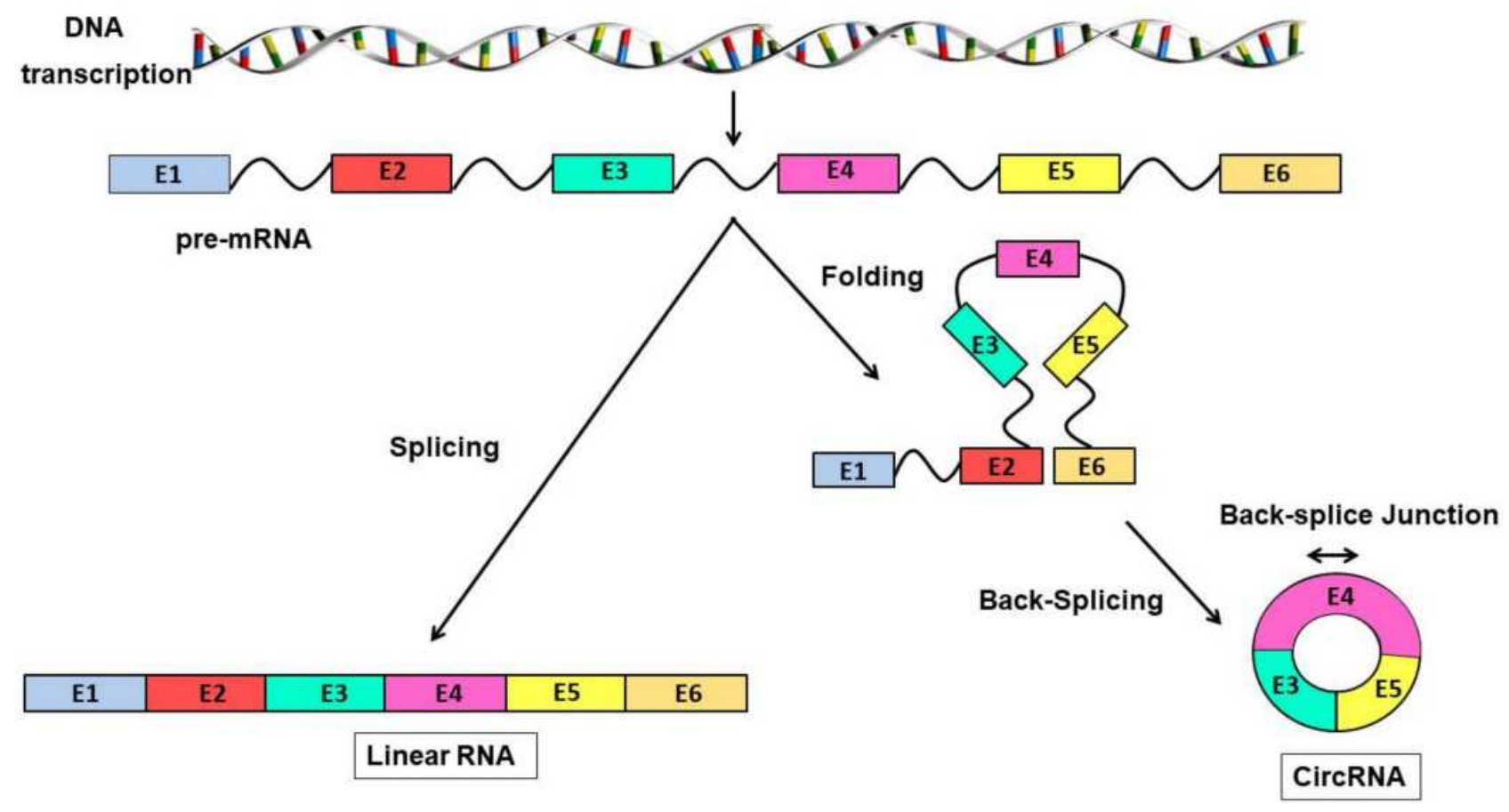

Figure 2 Formation of linear RNA and circRNAs DNA is transcribed into pre-mRNA, which in turn forms linear RNA or circRNAs in different ways. On the left, the premRNA is directly spliced to form linear RNA. On the right, the pre-mRNA, by folding and then splicing end to end, forms a circRNA, which is more stable.

Furthermore, in NSCLC, hsa_circ_0002130 targets miR498 to up-regulate GLUT1, hexokinase 2 (HK2), and lactate dehydrogenase A (LDHA), thereby regulating glycolysis and enhancing the resistance towards osimertinib, thus exerting a cancer-promoting effect. ${ }^{33}$ In colorectal cancer (CRC), the up-regulated circDENND4C directly targets and adsorbs miR-760, regulates GLUT1, and promotes glycolysis, growth, as well as metastasis of cells. ${ }^{34}$ In contrast, down-regulation of circDENND4C can inhibit glycolysis in CRC cells, and overexpression of GLUT1 can remedy this inhibition. The expression of circRNA_100290 in oral squamous cell carcinoma (OSCC) cells was significantly up-regulated, and GLUT1 was up-regulated through miR-378a to promote glycolysis, growth, and metastasis of cells. ${ }^{35}$

The study also found that in melanoma, the expression of circRNA (circ-ITCH) showed a downward trend, and the expression of GLUT1 as well as glucose uptake was promoted. However, overexpressed circ-ITCH can downregulate GLUT1 through ITCH, and inhibit glycolysis and cell proliferation, thus exerting anti-cancer effects. ${ }^{36}$ The over-expressed circRNA up-regulates GLUT and promotes the intracellular transport of glucose, playing a role in promoting cancer. It suggests that we can reduce the expression of certain circRNAs in tumors, down-regulate
GLUT as well as the absorption of glucose, and inhibit glycolysis to fight cancer through using the principles of molecular biology. Extensive research on the association of circRNAs- miRNAs and GLUT may help to elucidate the complex pathological mechanism of the Warburg effect.

\section{CircRNAs in Regulating Enzymes or Kinases of Glycolysis}

Ascending evidence reveals that the expression of enzymes or kinases is abnormal in some cancer, which is related to the reprogramming of cancer glucose metabolism. Carbohydrates are transported into the cell by an increased amount of GLUT to satisfy the tumor cell's need for glucose. But this is only the first step of the Warburg Effect, which then officially enters the glucose metabolism process. Glucose is converted to pyruvate by a variety of rate-limiting enzymes, such as hexokinase (HK), 6-phosphoglucose-1-kinase (PFK), and pyruvate kinase (PK). Lactic dehydrogenase A (LDHA) plays an important role in the final step of aerobic glycolysis, catalyzing the production of lactic acid from pyruvate. Recent literature has reported that in this process, some circRNAs can regulate glycolysis by targeting ENO1, PKM2, and LDHA. Finally, lactic acid is released into 


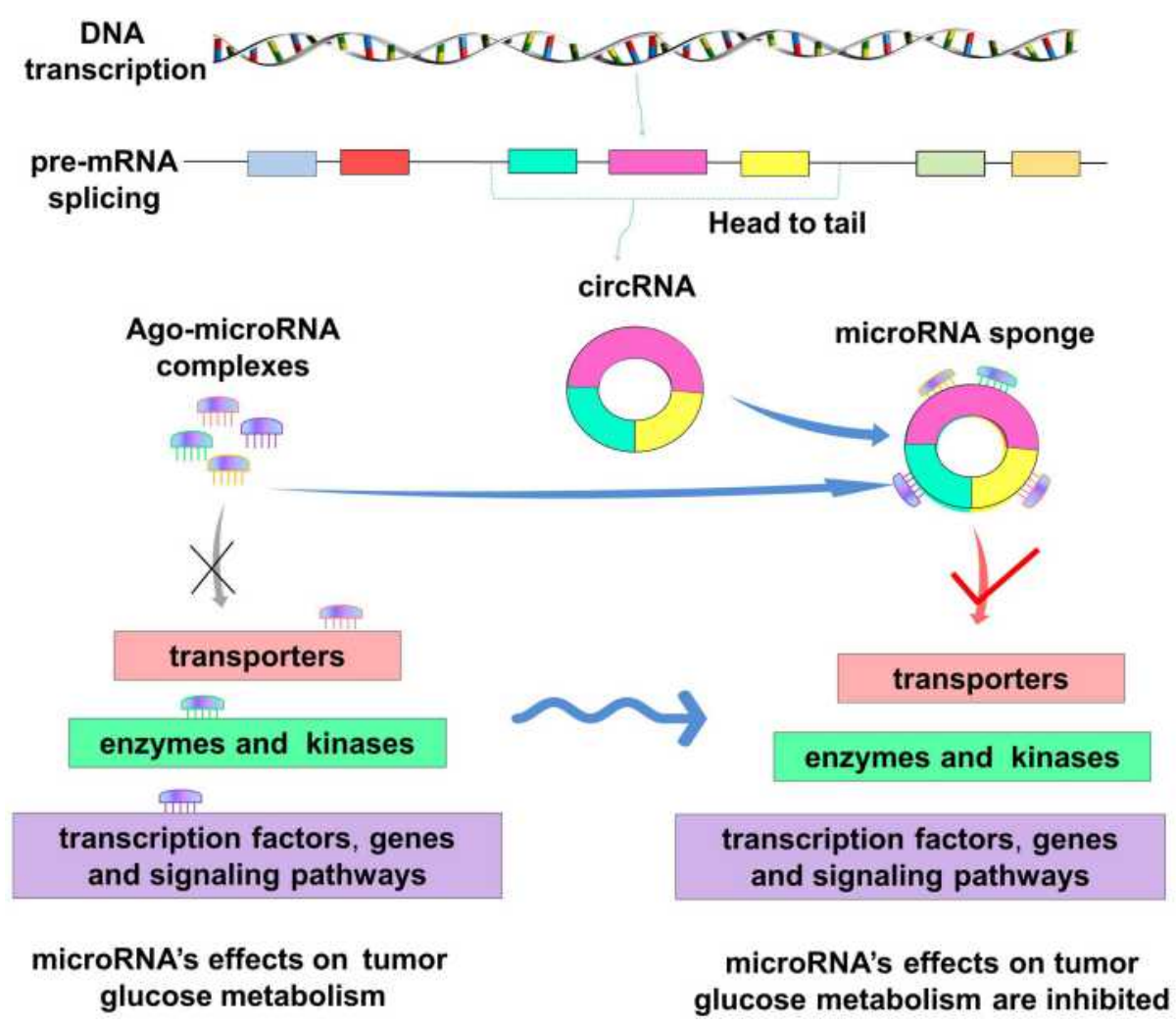

Figure 3 Mechanism of circRNA binding to microRNA. On the left, it shows the regulatory effects of microRNA on tumor glucose metabolism under normal conditions, and the ago-microRNA complexes can bind to transporters, enzymes, transcription factors or genes, signaling pathways and produce regulatory effects. On the right, in tumor cells, abnormal expression of circRNA formed by the head and tail splicing of pre-RNA is observed. The circRNAs bind to the ago-microRNA complexes as molecular sponges, making microRNA unable to bind to transporters, enzymes and kinases, transcription factors, genes and signaling pathways. Thus, the original regulatory effect of microRNA on tumor glucose metabolism is inhibited.

the extracellular matrix. Also, AKT1, as a protein kinase signaling pathway that triggers cancer, regulates the expression of TFs and affects cellular glycolysis by activating downstream cell growth regulators. In recent years, research on enzymes or kinases in the field of the reprogramming of cancer glucose metabolism has been increasing, and it was found that circRNAs have an important impact on the Warburg effect.

\section{ENOI}

Enolase 1 (ENO1), as a pivotal glycolytic enzyme, plays a regulatory role in the Warburg effect of tumor cells. In lung adenocarcinoma (LUAD), circ-ENO1 can increase the expression of ENO1 by targeting miR-22-3p. Silencing circ-ENO1 can inhibit cell glycolysis by reducing the activity of ENO1, and delay the uptake of glucose and the formation of lactic acid. Down-regulated circENO1 can reduce cell ATP levels, inhibit cell growth, migration, EMT, and induce apoptosis. ${ }^{37}$ The interaction mechanism between circ-ENO1 and ENO1 and their exact functions are worthy of further study.

\section{PKM2}

Pyruvate kinase (PK) is a key enzyme in both normal cellular glycometabolism, and abnormal cell glycolysis during tumorigenesis. The final step in the glycolysis reaction is the release of energy from the products pyruvate and ATP, driven by the previously produced ADP. In previous studies, ADP was obtained by the conversion of high-energy phosphoric acid by phosphoenolpyruvate catalyzed by PK, which is crucial for ATP generation.

Pyruvate kinase has four isoenzymes (PKXL, PKR, PKM1, and PKM2), and PKXL is expressed in the liver and kidney with strong gluconeogenesis. ${ }^{38} \mathrm{PKR}$ is mainly expressed in red blood cells. PKM1 is expressed in muscles and brains with rapid energy consumption and high oxygen consumption. PKM2 is expressed in embryonic cells, stem cells, and tumor cells with active nucleic acid syntheses. ${ }^{39}$ Tumor cells usually express PKM2, which plays an important role in cancer growth and carbohydrate metabolism. ${ }^{40}$ The dimeric form of PKM2 (restrictive) promotes the anabolism of intermediate biological macromolecules of glucose, while the tetrameric structure 
Table I CircRNAs in Cancer Glycolysis

\begin{tabular}{|c|c|c|c|c|c|}
\hline circRNA & Cancer Type & $\begin{array}{l}\text { Target } \\
\text { miRNA }\end{array}$ & $\begin{array}{l}\text { Downstream } \\
\text { Component }\end{array}$ & $\begin{array}{l}\text { Effect on } \\
\text { Glycolysis }\end{array}$ & Refs \\
\hline circ-Amotll & Breast cancer & - & $\begin{array}{l}\text { PDKI, AKTI, C-myc, } \\
\text { STAT3, AKT }\end{array}$ & Up & {$[30,72]$} \\
\hline $\operatorname{circARHGAPI0}$ & Non small cell lung cancer & miR-I50-5-p & GLUTI & Up & {$[31]$} \\
\hline hsa_circ_0002130 & Non small cell lung cancer & miR-498 & GLUTI, HK2, LDHA & $U_{p}$ & [33] \\
\hline circDENND4C & Colorectal cancer & miR-760, & GLUTI & Up & {$[34]$} \\
\hline circDENND4C & Breast cancer & $\begin{array}{l}\text { miR-200b, miR- } \\
200 c\end{array}$ & HIF-I $\alpha$, LDHA & Up & {$[61]$} \\
\hline circRNA_I00290 & Oral squamous cell carcinoma & miR-378a & GLUTI & Up & {$[35]$} \\
\hline circRNA_100290 & Colorectal cancer & $\begin{array}{l}\text { miR-29, miR- } \\
516 \mathrm{~b}\end{array}$ & $\begin{array}{l}\text { CDK6, RAS, Wnt/B- } \\
\text { catenin }\end{array}$ & Up & [93] \\
\hline circ-ITCH & Melanoma & - & GLUT & Up & [36] \\
\hline circ-ENOI & Lung adenocarcinoma & $m i R-22-3 p$ & ENOI & Down & {$[37]$} \\
\hline hsa_circ_0005963 & Colorectal cancer & miR-I22 & PKM2 & Up & {$[44]$} \\
\hline $\operatorname{circMAT2B}$ & Hepatocellular carcinoma & miR-338-3p & PKM2 & Up & {$[45]$} \\
\hline has_circRNA_403658 & Bladder cancer & - & LDHA & Up & [47] \\
\hline circMYC & Malignant melanoma & miR-I236 & LDHA & Up & {$[48]$} \\
\hline circ- 0033550 & Cervical cancer & miR-942-5p & AKTI, TGF- $\beta$ & Up & {$[53]$} \\
\hline circ-CFH & Glioma & $\operatorname{miR}-149$ & AKTI & Up & {$[54]$} \\
\hline circZFR & Hepatocellular carcinoma & miR-5II & AKTI & Up & [55] \\
\hline circPLEKHM3 & Ovarian cancer & miR-9 & AKTI & Down & [56] \\
\hline $\operatorname{circABCBI0}$ & Breast cancer & miR-223-3p & HIF-I, HK2, LDHA & Up & {$[63]$} \\
\hline circSLC25AI6 & Non small cell lung cancer & miR-488-3p & HIF-I $\alpha$, LDHA & Up & {$[64]$} \\
\hline circRNA_00I569 & Colorectal cancer & miR-145 & C-myc & Up & [70] \\
\hline $\operatorname{circBIRC6}$ & Colorectal cancer & $\begin{array}{l}\text { miR-I45, miR- } \\
34 a\end{array}$ & C-myc & Up & {$[7 \mid]$} \\
\hline circ CUXI & Neuroblastoma & - & CUXI, ENOI & Up & {$[70]$} \\
\hline circSEPT9 & Triple negative breast cancer & miR-637 & LIF/STAT3, p53 & Up & [7I] \\
\hline circRNA CBL. I I & Colorectal cancer & miR-6778-5p & YWHAE/p53 & Up & [77] \\
\hline circ-FOXMI & Melanoma & miR-143-3p & FLOT2 & $U_{p}$ & [78] \\
\hline $\operatorname{circRNA-MYLK}$ & Bladder cancer, Breast cancer & miR-29a-3p & RAS & Up & {$[80]$} \\
\hline Hsa_circ_0009910 & Hepatocellular carcinoma, Osteosarcoma & miR-449a & STAT3 & Up & [86] \\
\hline circRNA_100782 & Pancreatic ductal adenocarcinoma & miR-124 & STAT3 & Up & {$[87]$} \\
\hline $\operatorname{circNRIPI}$ & Gastric cancer & miR-I49-5p & AKTI & Up & [91] \\
\hline circ_0067934 & Thyroid cancer & miR-I 324 & $\mathrm{PI} 3 \mathrm{~K} / \mathrm{AKT}, \mathrm{Wnt} / \beta$-catenin & Up & [92] \\
\hline
\end{tabular}


Table I (Continued).

\begin{tabular}{|c|c|c|c|c|c|}
\hline circRNA & Cancer Type & $\begin{array}{l}\text { Target } \\
\text { miRNA }\end{array}$ & $\begin{array}{l}\text { Downstream } \\
\text { Component }\end{array}$ & $\begin{array}{l}\text { Effect on } \\
\text { Glycolysis }\end{array}$ & Refs \\
\hline circRNA_I0380I & $\begin{array}{l}\text { Osteosarcoma, Esophageal squamous cell } \\
\text { carcinoma }\end{array}$ & $\begin{array}{l}\text { miR-370, miR- } \\
877\end{array}$ & PI3K/AKT, HIF-I & $U_{p}$ & {$[94]$} \\
\hline circRNA_000I72I & Osteosarcoma & miR-372-3p & MAPK7, PI3K/Akt & $U_{p}$ & {$[95]$} \\
\hline $\begin{array}{l}\text { circRNA9953-PKN2, } \\
\text { etc. }\end{array}$ & Esophageal squamous cell carcinoma & - & $\mathrm{PI} 3 \mathrm{~K} / \mathrm{Akt}$ & $U_{p}$ & {$[97]$} \\
\hline circ-ITCH & Papillary thyroid cancer & $\operatorname{miR}-22-3 p$ & $\mathrm{CBL}$ & Down & {$[101]$} \\
\hline circRNA_10217I & Papillary thyroid carcinoma & - & Wnt $/ \beta$-catenin & Down & {$[100]$} \\
\hline circ_0006427 & Lung adenocarcinoma & $\operatorname{miR}-6783-3 p$ & Wnt $/ \beta$-catenin & Down & {$[103]$} \\
\hline circCDRlas & Non small cell lung cancer & $\operatorname{miR}-219 a-5 p$ & SOX5, Wnt/B-catenin & $U_{p}$ & {$[104]$} \\
\hline circCDRlas & Nasopharyngeal carcinoma & miR-7-5p & $\mathrm{E} 2 \mathrm{~F} 3, \mathrm{Wnt} / \beta$-catenin & Down & {$[105]$} \\
\hline $\operatorname{circZKSCANI}$ & Hepatocellular carcinoma & - & MYB, PDKI & Down & {$[106]$} \\
\hline circDCUNID4 & Lung adenocarcinoma & - & $\mathrm{HuR} / T X N I P$ & Down & {$[107]$} \\
\hline $\operatorname{circFNDC3B}$ & Colorectal cancer & - & circFNDC3B-2 I8aa & Down & {$[108]$} \\
\hline
\end{tabular}

Note: Means not mentioned in the paper.

(activated) promotes the OXPHOS of glucose and provides energy for cells. ${ }^{41}$ The overexpression of PKM2 and the transformation between dimers and tetramers of PKM2 enable tumor cells to survive in various oxygen as well as nutrient environments. ${ }^{42}$

When PKM2 is overexpressed, a large amount of glucose will be converted into lactic acid, and the glycolysis rate will be increased to generate ATP quickly. ${ }^{43}$ In CRC, hsa_circ_0005963 (ciRS-122) exosomes are transported from oxaliplatin-resistant cells to sensitive cells. ${ }^{44}$ In oxaliplatin-resistant cells, the reduced ciRS-122 up-regulates the expression of PKM2 by targeting miR-122, thus promoting aerobic glycolysis, ATP production, and the development of CRC. In hepatocellular carcinoma (HCC), circMAT2B promotes cancer by exciting the circMAT2B/ miR-338-3p/PKM2 axis, thereby stimulating glycolysis. ${ }^{45}$ CircRNA regulates the glucose metabolism of CRC and HCC through PKM2, so the function of circRNAs related to PKM2 in tumor progression deserves further study.

\section{LDHA}

LDHA catalyzes the formation of lactic acid from pyruvate, which is the last step of aerobic glycolysis. ${ }^{46}$ CircRNAs in tumor cells can regulate glycolysis by regulating LDHA, thereby affecting tumor cell proliferation and apoptosis. For example, in bladder cancer (BC) cells under hypoxic conditions, the up-regulated has_circRNA_403658 can promote aerobic glycolysis by regulating the expression of LDHA, which indicates a poor prognosis. Has_circRNA_403658 is induced by excessive HIF1 and is encoded by its host gene ZNF292. On the contrary, when the expression of has_circRNA_403658 was knocked down, the expression of LDHA was significantly down-regulated, and LDH (lactate dehydrogenase) activity, glucose absorption, lactate production as well as ATP production were reduced. ${ }^{47}$ Besides, in Mel-CV cells and human melanoma cells, the significantly increased circMYC promotes the glucose metabolism of tumor cells through the circMYC/miR1236/LDHA axis and has a cancer-promoting effect. ${ }^{48}$

\section{AKTI}

As a protein kinase that triggers cancer, AKT1 is a cell growth regulator and one of the most critical kinases in the tumor growth pathway. ${ }^{49,50}$ AKT1 regulates cell glucose metabolism by up-regulating metabolism-related TFs, thus affecting cell proliferation and apoptosis. ${ }^{51,52}$

Plenty of evidence shows that in cervical cancer (CC), the expression of circ-0033550 (circ-AKT1) and AKT1 are significantly increased. Circ-AKT1 targets miR-942- 
$5 p$ and up-regulates transforming growth factor $\beta$ (TGF- $\beta$ ) to promote the epithelial-mesenchymal transition (EMT), glycolysis, growth, and metastasis of $\mathrm{CC}^{53}$ In glioma tissue, circ-CFH was significantly up-regulated. In U251 and U373 cells, circ-CFH is a regulator of glucose metabolism and functions through the circ-CFH/miR-149/ AKT1 axis. ${ }^{54}$ In HCC, the up-regulated circZFR targets miR-511 and up-regulates AKT1, thereby cascading activation of proliferation-related proteins (c-Myc, Survivin, cyclin D1, and Bcl-2). CircZFR promotes HCC glycolysis through the circZFR/miR-511/AKT1 axis. ${ }^{55}$

Studies have found certain circRNA that is downregulated in tumors can inhibit glycolysis, proliferation, and migration of tumor cells by down-regulating AKT1. In ovarian cancer cells, the significantly reduced circPLEKHM3 enhances the endogenous inhibitory effects of BRCA1, DNAJB6, and KLF4 by targeting miR-9 and inactivates AKT1 signaling, which is closely related to poor prognosis. (DNAJB6 can bind to the phosphorylation site of AKT1. KLF4 participates in the transcriptional regulation of AKT1 through catenin. BRCA1 has the ability to inhibit the activation of AKT1). CircPLEKHM3 inhibits the glycolysis and progression of ovarian cancer by regulating the circPLEKHM3/miR-9/ BRCA1/DNAJB6a/KLF4/AKT1 axis, which is expected to become a clinical therapeutic target for ovarian cancer. ${ }^{56}$ Therefore, the in-depth study of the detailed mechanisms of $\mathrm{CC}$ and ovarian cancer related to AKT1 may bring hope to the treatment of female cancer. However, it is not clear which circRNA can play a more dominating role and what is the association between these circRNAs in the reprogramming of cancer glucose metabolism.

\section{CircRNAs in Regulating Transcription Factors, Genes, and Signaling Pathways of Glycolysis}

Numerous studies have found that distinctive TFs also have an impact on the Warburg effect. As one of the pivotal members of ncRNAs, circRNAs serve a regulative function and control the mutative expression of TFs. The circRNAs target miRNAs and further modulate the target TFs of miRNAs, such as HIF-1, C-myc, CUX1, p53, FLOT2, RAS, and STAT3. Besides, the regulatory network of Warburg Effect also involves classical signaling pathways related to circRNAs, such as PI3K/Akt and $\mathrm{Wnt} / \beta$-catenin. These TFs, signaling pathways, and
circRNAs interweave to form a regulatory network of Warburg effect, which lays a foundation for further research on the mechanism of glucose metabolism in tumor cells. Thus, it is of vital importance to keep a watchful eye on representative TFs, signaling pathways as well as the associated circRNAs.

\section{HIF-I}

The Warburg effect is closely related to the hypoxic microenvironment. ${ }^{57}$ In response to hypoxic conditions, oncogenes in solid tumors are activated, and the transcription of genes and enzymes related to glucose metabolism is promoted (HIF-1, monocarboxylate transporter, PKM, and $\mathrm{HK}$ are up-regulated), which in turn promotes Glycolysis. ${ }^{58}$ Besides, the up-regulation of HIF-1 promotes the formation rate of lactic acid by up-regulating PDK1. ${ }^{59}$ In human umbilical cord vascular endothelial cells (HUVEC), the over-expressed circ_0010729 promotes glycolysis and cell growth, by targeting miR-186 and up-regulating HIF-1 $\alpha$, especially in a hypoxic microenvironment. As a result of positive feedback, the rapid growth of HUVECs can accelerate the intracellular transport of glucose. ${ }^{60}$

Under hypoxic conditions, HIF-1 $\alpha$ can up-regulate circDENND4C in breast cancer cells. CircDENND4C can target miR-200b and miR-200c, and then regulate the downstream effectors (LDHA and sirtuin 2) of miRNA to inhibit tumor glycolysis and promote cell proliferation. ${ }^{61}$ Besides, in breast cancer tissues, circABCB10 and PFN2 increased, while miR-223-3p decreased. Silencing circABCB10 inhibits cell glycolysis and proliferation by regulating miR-223-3p, and reduces the insulin resistance (IR) of breast cancer cells, which may be related to HIF-1, HK2, and LDHA. ${ }^{62}$ CircSLC25A16 can promote glycolysis of NSCLC cells and affect cell metabolisms, such as promoting glucose uptake, accelerating lactic acid production, enhancing ATP production capacity as well as extracellular acidification rate (ECAR). ${ }^{63}$ CircSLC25A16 promotes LDHA transcription and promotes glycolysis through the circSLC25A16/miR-488-3p/HIF-1 $\alpha$ axis. ${ }^{64}$ It is necessary to pay close attention to the circRNAs related to HIF-1 and explore the treatment of breast cancer combined with HIF.

\section{C-Myc}

As one of the important carcinogenic $\mathrm{TFs},{ }^{49} \mathrm{c}$-myc is an important regulator of cell metabolism and can promote the Warburg effect. ${ }^{65,66}$ Overexpressed HIF-1 and c-myc 
cooperate to promote glycolysis in tumor cells by upregulating the key components of glycolysis (GLUT1, HK2, and PDK1). ${ }^{59,67}$

Furthermore, in CRC, both circRNA_001569 and circBIRC6 can target miR-145. ${ }^{68,69}$ Certain circRNAs can target several miRNAs. ${ }^{70}$ For example, in addition to miR-145, circBIRC6 also targets miR-34a, which is a miRNA that can inhibit c-myc. ${ }^{69}$ The oncogene c-myc can also be regulated by several circRNAs. ${ }^{71}$ In addition to circBIRC6, circ-Amotl1 can also inhibit c-myc nuclear translocation mutations, and can also affect common signal transduction activator transcription 3 (STAT3) and $\mathrm{AKT}^{72}$ In other words, circ-Amotl1 can enhance aerobic glycolysis to promote tumor development, rather than through the ceRNA-miRNA mechanism. Circ-FBXW7 encodes the protein FBXW7-185aa, which can interact with the deubiquitinating enzyme USP28 to directly promote the degradation of c-myc and enhance the Warburg effect. $^{73}$ Hence, the not entirely cleared interactive mechanism between circRNA and c-myc is worth studying.

\section{CUXI}

Produced during the hydrolysis of p200 CUX1 protein, the TF p110CUX1 can promote the expression of phosphoglycerate kinase 1 (PGK1), glucose 6 phosphate isomerase (G6PI), and ENO1. ${ }^{70}$ Besides, circ CUX1 can bind to EWS RNA binding protein 1 (EWSR1) and promote the interaction between it and MYC-related zinc finger protein (MAZ). The transactivation of zinc finger protein affects the transcription of CUX1 and tumor progression. Overexpressed circ CUX1 can promote glucose uptake and lactate and ATP production through the circ CUX1/EWSR1/ MAZ axis. Knockout of circ CUX1 can significantly inhibit tumor glycolysis, growth, invasion, migration and metastasis of neuroblastoma (NB) cells. ${ }^{70} \mathrm{NB}$ is one of the extracranial malignancies with a high incidence in children, so it is necessary to check the exact function of this signaling pathway to obtain potential treatment targets.

\section{P53}

Many important TFs can regulate tumor metabolism. ${ }^{74}$ In addition to hypoxia-inducible factor 1 (HIF1) and the oncogene c-myc, the tumor suppressor gene P53 also plays a key role in the regulation of metabolism. ${ }^{66,75}$ Matoba's research clearly shows that the tumor suppressor gene p53 can regulate tumor glucose metabolism, mitochondrial activity, and oxygen consumption, thereby ensuring the balance between mitochondrial respiration and glycolysis. $^{76}$

CircRNA can regulate tumor metabolism through p53. For example, circSEPT9, which is significantly upregulated in triple-negative breast cancer (TNBC), can bind miR-637 to regulate leukemia inhibitory factor (LIF), p53, and GLUTs to promote glycolysis. CircSEPT9 can also activate the LIF/STAT3 signaling pathway and promote the growth and metastasis of TNBC, which is closely related to advanced cancer and suggests a poor prognosis. $^{71}$ Moreover, circRNA CBL.11 can regulate the glucose metabolism of CRC cells and tumor proliferation through the CBL.11/miR-6778-5p/YWHAE/p53 signaling pathway. ${ }^{77}$ As mentioned above, the regulation mechanism of P53 and circRNAs in glucose metabolism is worthy of further study, which will help to find cancer treatments.

\section{FLOT2}

As a functional and stable lipid raft, Flotillin 2 (FLOT2) exists in the cell membrane and is highly expressed in a variety of malignant tumors. FLOT2 gene affects tumor metabolism, proliferation, and metastasis through $\mathrm{NF}-\kappa \mathrm{B}$, $\mathrm{PI} 3 \mathrm{~K} / \mathrm{Akt}, \mathrm{Wnt} / \beta$-catenin, and $\mathrm{p} 53$. These pathways are regulated by ceRNAs and various signaling pathways.

In melanoma, circ-FOXM1 up-regulates FLOT2 by targeting miR-143-3p and promotes glycolysis and cell proliferation activity through the circ-FOXM1/miR-1433p/FLOT2 axis. Silencing circ-FOXM1 can inhibit cell glycolysis and reduce cell proliferation and metastasis, which is of great significance in the clinical treatment of melanoma. ${ }^{78}$ Melanoma usually has a poor prognosis, and the function of the above-mentioned signal axis in tumor metabolism and development is worthy of further study.

\section{RAS}

In NSCLC, CRC, and prostate cancer (PCa), KRAS is mutated, which upregulates GLUT1 and glycolytic enzymes, and promotes the Warburg effect. ${ }^{78}$ In BC, abnormally expressed circRNA-MYLK regulates RAS through the Raf/MEK/ERK signal axis. circRNAMYLK can activate vascular endothelial growth factor (VEGF), increase glucose uptake, and bind miR-29a by targeting. $^{79}$ Furthermore, in breast cancer, both circRNA-MYLK and long non-coding RNA H19 (lncRNA H19) can be used as ceRNA to bind miR$29 a-3 p .{ }^{80,81}$ In liver cancer, circRNA-MYLK can upregulate PKM2 by combining with miR-29a as 'well as up-regulating c-myc and HRAS. $^{82}$ In addition, 
circRNA_100290 binds to miR-29, which is beneficial to enhance the expression of RAS. In order to find effective therapeutic targets, RAS and its related circRNA should be studied to understand the mechanism of tumor metabolism and progression.

\section{STAT3}

Under the stimulation of circ-Amotl1, the expression of STAT3 increases, which stimulates tumorigenesis by inducing inflammation. STAT3 can also directly increase HK2 in breast cancer cells and promote cell proliferation. ${ }^{83}$ In HCC, overexpressed hsa_circ_0009910 binds to miR-449a to up-regulate $\mathrm{Bcl}-2$ and interleukin 6 receptor (IL6R), which is associated with STAT3, but silencing circRNA can reduce the phosphorylation level of STAT3. ${ }^{84}$ In osteosarcoma, Hsa_circ_0009910 binds to miR-449a, upregulates STAT3, and promotes glycolysis. ${ }^{85}$ Besides, in pancreatic ductal adenocarcinoma (PDAC), the significantly up-regulated circRNA_100782 binds to miR-124, activates the Janus kinase 2 (JAK2) family through IL6R, then activates STAT3, promotes carbohydrate metabolism and proliferation of tumors. ${ }^{86}$ Silencing circRNA_100782 will inhibit the metabolism, proliferation, and colony formation of human pancreatic adenocarcinoma cell 3 (BxPC3) in situ through the IL6/Jak2/STAT3 signaling pathway. ${ }^{87}$ More research should be carried out to reveal the circRNAs related to STAT3 and find effective therapeutic targets.

\section{$\mathrm{PI3K} / \mathrm{Akt}$}

Formed by phosphatidylinositol 3-kinase (PI3K) and Akt together, the PI3K/Akt signaling pathway can enhance aerobic glycolysis and is a regulator of tumor glucose metabolism. PI3K/Akt signaling pathway can promote glucose absorption and stimulate glycogen synthesis, by enhancing the antioxidant activity of HIF-1 $\alpha$ and upregulating phosphofructokinase 2 (PFK2) and $\mathrm{HK} 2{ }^{88}$ At the downstream of Akt, mTOR complex1 can promote the phosphorylation of elf4e binding protein and up-regulate PFK2, HK2, and GLUT1, thereby regulating the glucose metabolism of tumor cells. ${ }^{89,90}$ For example, in NSCLC, miR-124 can reduce HK2 and GLUT1 by targeting AKT1 and AKT2, thereby weakening the Warburg effect. ${ }^{28}$

In gastric cancer, the expression of circNRIP1 is upregulated, which promotes glucose uptake and lactate formation through miR-149-5p/AKT1. ${ }^{91}$ In thyroid cancer, circ_0067934 promotes cell glycolysis, proliferation, and metastasis through circ_0067934/miR-1324/FZD5/Wnt/ $\beta$ - catenin axis. On the contrary, silencing circ_0067934 will slow down cell metabolism through the PI3K/AKT signaling pathway. $^{92}$ Besides, in OSCC, circRNA_100290 down-regulates cyclin-dependent kinase 6 (CDK6) by targeting miR-29. ${ }^{93}$ In addition, circRNA_103801, which is abnormally expressed in osteosarcoma and esophageal squamous cell carcinoma (ESCC), can target and bind miR-370 and miR-877, thereby regulating cellular glucose metabolism by regulating HIF-1 and PI3K/AKT pathways. $^{94}$ Also, circRNA_100876 inhibits glycolysis and cell proliferation by targeting miR-136. ${ }^{32}$ Moreover, the expression of circRNA_0001721 is significantly upregulated, which can combine with miR-569 and miR-599 to play a regulatory function. In osteosarcoma, circ_0001721 targets miR-372-3p to up-regulate MAPK7 and promote glycolysis. Knockout of Circ_0001721 can inhibit glycolysis, proliferation, and migration of osteosarcoma cells. ${ }^{95}$ Under the regulation of PI3K/Akt signaling pathway, the phosphorylation level of glycogen synthase kinase $3 \beta$ (GSK-3 $\beta$ ) decreases, the activity of Akt and ubiquitin ligase changes, and insulin-like growth factor I (IGF-1) Induces the expression of BNIP3, thus affecting the cell cycle and promoting tumor growth. ${ }^{96}$

In addition, the abnormal expression of circRNA9953PKN2， circRNA3706-LAMA3， circRNA5431-PRKAA1, circRNA7681-FN1, circRNA8507-LAMC2, circRNA3703LAMA3, circRNA3341-HSP90AA1, circRNA4628-EGFR, circRNA7574-ITGA6 and circRNA8506-LAMC1 were detected in ESCC. ${ }^{97} \mathrm{PI} 3 \mathrm{~K} / \mathrm{Akt}$ signaling pathway can promote glycolysis, affect energy metabolism, regulate tricarboxylic acid cycle (TAC) and mitochondrial function, which are closely related to various enzymes and signaling molecules. The PI3K/Akt signaling pathway has many potential circRNA targets, and the in-depth mechanism is still elusive and needs to be explored. Moreover, the authors believed that those circRNAs might be highly effective biomarkers for early diagnosis of ESCC.

\section{$\mathrm{Wnt} / \beta$-Catenin}

As we all know, as one of the most important signal pathways in tumor cell metabolism, the $\mathrm{Wnt} / \beta$-catenin signaling pathway can up-regulate the transcription levels of PDK1 and MCT1 to enhance the Warburg effect. ${ }^{98,99}$ CircRNA_102171 is highly expressed in papillary thyroid carcinoma (PTC) and targets CTNNBIP1 to inhibit its downstream $\quad W n t / \beta$-catenin/TCF3/TCF4/LEF1 signal axis. ${ }^{100}$ In PTC, circ-ITCH can also up-regulate CBL to regulate tumor glycolysis by targeting miR-22-3p. As the 
E3 ligase of nuclear $\beta$-catenin, CBL inhibits the Warburg effect in PTC by degrading $\beta$-catenin. ${ }^{101}$ Moreover, both circ_0067934 and circRNA_100290 can promote the Wnt/ $\beta$-catenin pathway by targeting miR-1324 and miR-516b respectively. ${ }^{100,102}$ In LUAD, circ_0006427 can target miR-6783-3p, leading to inactivation of the $\mathrm{Wnt} / \beta-$ catenin pathway. ${ }^{103}$ In NSCLC cells, the circCDR1as/ miR-219a-5p/SOX5 axis may target the $\mathrm{Wnt} / \beta$-catenin pathway and regulate the Warburg effect. ${ }^{104}$ In nasopharyngeal carcinoma (NPC), circCDR1as can increase the glucose consumption and the production of lactic acid by targeting miR-7-5p and upregulating E2F3. On the contrary, when circCDRlas is silenced, the $\mathrm{Wnt} / \beta$-catenin pathway significantly inhibits the metabolism of HK1 and HONE1 cells (NPC line). ${ }^{105}$ All in all, circRNAs that regulate tumor cell metabolism of LUAD and NPC are closely related to the $\mathrm{Wnt} / \beta$-catenin pathway. However, the current research is still in the initial stage, and the function of circRNAs associated with $\mathrm{Wnt} / \beta$ catenin pathway needs further exploration, especially in cancer glucose metabolism.

\section{Other Biological Functions of circRNAs Associated with Cancer Glucose Metabolism}

As introduced above, many scholars have conducted a lot of research on the function and mechanism of circRNA in recent years. The main biological functions of circRNA mainly include the following four types. (i) circRNA acts as a molecular sponge of miRNA. CircRNA binds to miRNA through MRE, thereby interfering with gene silencing caused by the miRNA-mRNA axis. ${ }^{14}$ It is true for most of the examples discussed above. (ii) CircRNA plays a biological role by regulating gene transcription. CircRNA-ENO1 promotes glycolysis and tumor progression of LUAD by up-regulating its host gene ENO1. ${ }^{37}$ Besides, the ZKSCAN1 gene and its related circRNA (circZKSCAN1) inhibit the metabolism, growth, migration, and invasion of HCC cells through different signal transduction pathways such as COL3A1, CDH5, MYB, PDK1 as well as BCL2. ${ }^{106}$ (iii) CircRNA can also be combined with RBP to regulate a variety of biological activities, including cell proliferation, differentiation, movement, apoptosis and aging. In LUAD, circDCUN1D4 interacts with a kind of RBP called human antigen $\mathrm{R}(\mathrm{HuR})$ to stabilize the expression of thioredoxin-interacting protein (TXNIP) to inhibit tumor metastasis and glycolysis, indicating the formation of the circDCUN1D4/HuR/TXNIP RNA-protein ternary complex. ${ }^{107}$ (iv) CircRNA is involved in protein translation. At first, it was thought that circRNA did not have the protein-coding function, but recently it was discovered that some circRNAs have highly conserved internal ribosome entry sites (IRES) with translation function. For example, circFNDC3B-218aa, a novel protein encoded by circFNDC3B, can affect glucose, thereby inhibiting the progression of CRC and EMT. ${ }^{108}$ CircRNAs mainly regulate tumor metabolism through the ceRNA-miRNA pathway, which has important biological significance for targeted tumor therapy. At present, the latter three biological functions have not been studied much in the regulation of tumor metabolism, and they are worthy of in-depth exploration.

\section{Cancer Therapy Associated with circRNAs and Glucose Metabolism}

Several up-to-date studies have shown that in BC, lung cancer, glioma as well as CRC, anticancer drugs can regulate cell glycolysis by regulating circRNA-miRNA axes to inhibit tumor growth and proliferation. ${ }^{109}$ As a potential therapeutic drug for $\mathrm{BC}$, puerarin can inhibit glycolysis to regulate the circ_0020394/miR-328-3p/ NRBP1 axis to weaken cell viability, inhibit tumorigenesis, cell growth, and migration in vivo, and promote apoptosis of BC cells. ${ }^{110}$ Besides, propofol therapy can inhibit the aerobic glycolysis, growth, proliferation, migration, and invasion of lung cancer cells by regulating the circTADA2A/miR-455-3p/FOXM1 axis, which has clinical significance in preventing the development of lung cancer. ${ }^{111}$ Similarly, in glioma tissues and cells, sevoflurane achieves the purpose of suppressing cancer through the circ_0002755/miR-628-5p/MAGT1 axis. Sevoflurane reduces the consumption of glucose and the production of lactic acid through the signal axis, thus inhibiting tumor growth, migration as well as promoting cell apoptosis in the body. ${ }^{112}$ Besides, lidocaine inhibits the aerobic glycolysis of CRC cells through the circHOMER1/miR-138-5p/ HEY1 axis, thereby promoting cell apoptosis in vitro and inhibiting tumor growth in vivo. ${ }^{113}$ These reports have laid the foundation for targeted cancer therapy, but the regulatory network of circRNA and glucose metabolism still needs to be further enriched in the years ahead to obtain effective targeted therapy methods for malignant tumors. 


\section{Conclusions and Perspectives}

Extensive exploration in the field of circRNA research has brought us a lot of new insights into cancer glucose metabolism. Mounting evidence has revealed that abnormally expressed circRNAs may trigger the Warburg effect as well as the development of malignant tumors, thus we highlight the role of circRNAs in cancer carbohydrate metabolism regulatory networks and how they contribute to cancer malignancy. In view of existing reports, we reviewed molecular mechanisms of circRNAs in regulating tumor glycolysis by regulating transporters, enzymes, kinases, TFs, genes, and glycolysis signaling pathways (Figure 4). The regulatory mechanisms mediated by circRNAs in tumor metabolism can be divided into the following 4 types. (i) As ceRNAs, circRNAs competitively sponge miRNAs and regulate tumor glycolysis related signal axes. (ii) CircRNAs can interact with RNA polymerase II or splicing factors to regulate gene transcription and affect tumor glycolysis. (iii) CircRNAs may also bind to RBPs to regulate tumor metabolism by competing with the RBP substrates. (iv) CircRNAs can encode proteins to affect tumor glucose metabolism. CircRNAs have other functions that may be involved in regulating tumor glucose metabolism. CircRNAs can substitute their linear counterparts. Circ-FBXW7 attracted USP28 as a substitution of its linear counterpart to reduce the halflife of c-Myc by antagonizing the stability of c-Myc. And the above process inhibits cell proliferation and cell cycle

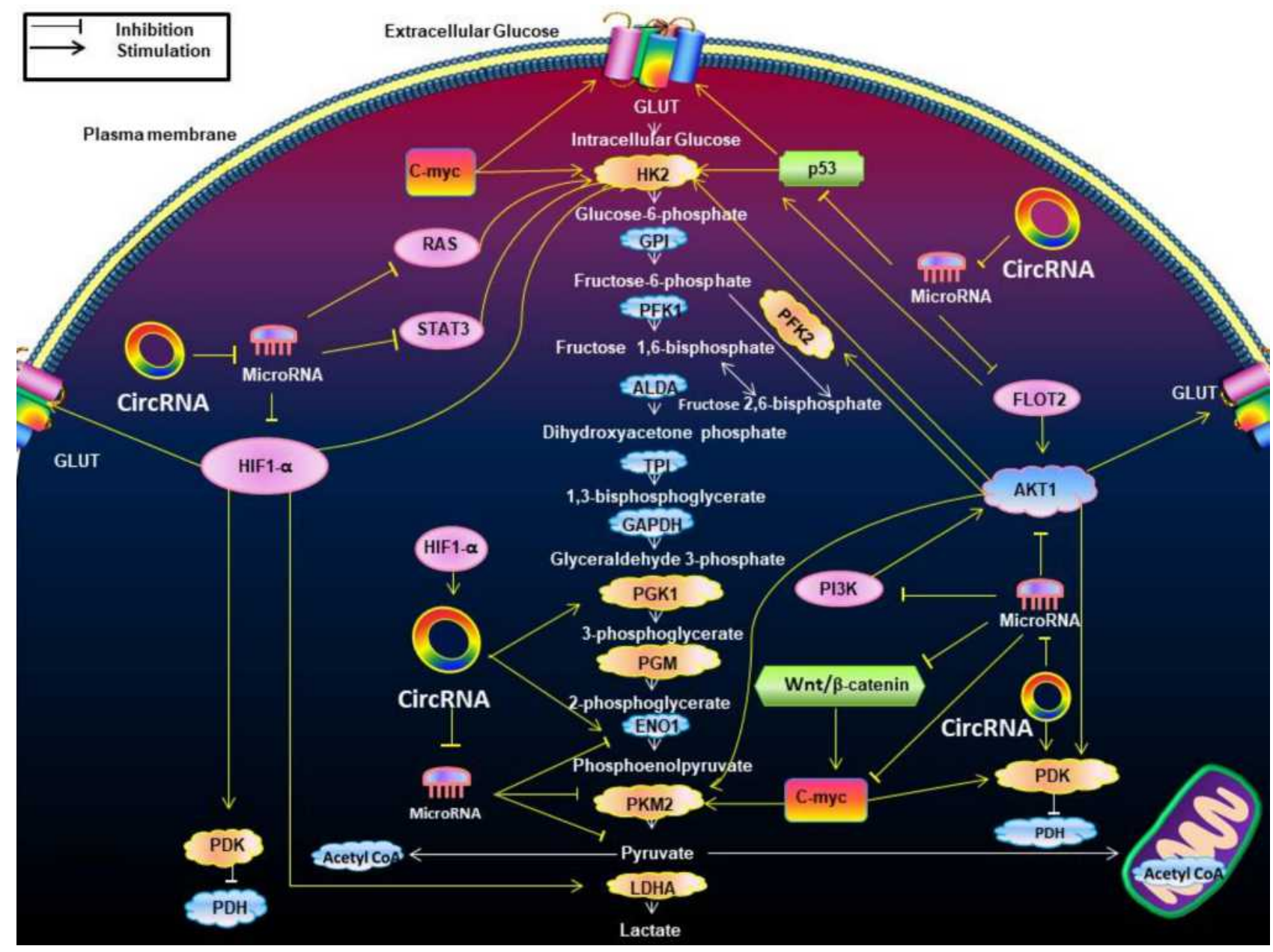

Figure 4 The effects of circRNAs on the Warburg effect-associated signaling pathways. This figure indicates the effects of circRNAs on the Warburg effect-associated signaling pathways. It shows how circRNAs interact with these metabolic pathways by enzymes and transporters associated with the Warburg effect. The majority of circRNAs can antagonize microRNAs to indirectly produce effects. HIFI- $\alpha$ regulates GLUT, HK2, PDK, LDHA. The PI3K/Akt pathway promotes glucose GLUT, HK2, PFK2, pyruvate kinase M2 (PKM2), and PDK. The change of RAS pathway up-regulated HK2. P53 up-regulated GLUT, HK2. The STAT3 pathway promotes HK2. Wnt/3-catenin pathway up-regulates PDK transcription while promoting c-myc. The positive relationships are shown by arrows, and the negative relationships are shown by short dashes. Abbreviations: GLUT, glutamate dehydrogenase; HK2, hexokinase 2; GPI, glycosyl phosphatidyl inositol; PFKI, phosphate fructose kinase I; ALDA, aldolase A; TPI, triosephosphate isomerase; GAPDH, glyceraldehyde phosphate dehydrogenase; PGKI, phosphoglycerate kinase I; PGM, phosphoglucomutase; ENOI, enolase I; PKM2, pyruvate kinase isozymes M2; LDHA, lactate dehydrogenase A; PDK, pyruvate dehydrogenase kinase; PDH, pyruvate dehydrogenase. 
Table 2 The Regulatory Mechanism of circRNA

\begin{tabular}{|l|l|l|}
\hline $\begin{array}{l}\text { Regulatory } \\
\text { Mechanism }\end{array}$ & circRNA & Refs \\
\hline $\begin{array}{l}\text { circRNAs } \\
\text { sponge } \\
\text { miRNAs as } \\
\text { ceRNAs }\end{array}$ & $\begin{array}{l}\text { circMAT2B, } \\
\text { circMYC, circ- } \\
\text { CFH, circZFR, } \\
\text { circPLEKHM3, } \\
\text { circSLC25AI6, } \\
\text { circ CUXI, } \\
\text { circ-FOXMI, } \\
\text { circ_0067934, } \\
\text { circCDRIas, } \\
\text { etc. }\end{array}$ & \\
\hline $\begin{array}{l}\text { circRNAs } \\
\text { regulate gene } \\
\text { transcription }\end{array}$ & $\begin{array}{l}\text { circRNA-56,64,70,78,92,104, 105] } \\
\text { circZKSCANI }\end{array}$ & {$[37,106]$} \\
\hline $\begin{array}{l}\text { circRNAs } \\
\text { combine } \\
\text { with RBPs }\end{array}$ & circDCUNID4 & {$[107]$} \\
\hline $\begin{array}{l}\text { circRNAs } \\
\text { encode } \\
\text { proteins }\end{array}$ & circFNDC3B \\
\hline $\begin{array}{l}\text { circRNAs } \\
\text { substitute } \\
\text { their linear } \\
\text { counterparts }\end{array}$ & circ-FBXW7 \\
\hline $\begin{array}{l}\text { ircRNAs } \\
\text { translocation }\end{array}$ & {$[73]$} \\
\hline
\end{tabular}

acceleration. ${ }^{73}$ Moreover, circRNAs can induce nuclear translocation. Circ-Amotll induces the nuclear translocation of PDK1, AKT1, and c-myc, and then changes the glycolysis process by influencing downstream effectors. ${ }^{30}$ These approaches have opened up a broad new world for the study of the biological functions of circRNA in cells and established a bridge with new tumor regulatory systems (Table 2).

It is worth noting that, the same circRNA may exert diametrically opposite functions through different signal axes in different types of tumor tissues. CircCDR1as promotes tumor glycolysis through miR-219a-5p/SOX5/Wnt/ $\beta$-catenin in NSCC, but inhibits tumor glycolysis through miR-7-5p/E2F3/Wnt/ $/$-catenin in NPC. Circ-ITCH promotes glycolysis of melanoma by regulating GLUT, but inhibits glycolysis of papillary thyroid cancer through miR-22-3p/CBL. However, the study of the opposite functions of the same circRNA is still in their infancy, so a large number of experiments are still required. It is clear that the variation of circRNA levels in tumor tissues is a universal phenomenon, and the efficient influence of many circRNAs acting in concert may be needed to enact a function. If certain circRNAs turn out to be detrimental or beneficial to the Warburg effect, then it will become lucrative to investigate the feasibility of therapeutic interventions through manipulating circRNAs. Now we put forward several research interests that have not been studied thoroughly, hoping to provide inspiration for indepth exploration in circRNAs studies.

\section{Circulating circRNAs and Cancer Glucose Metabolism}

Through greater recognition of circRNAs and powerful high-throughput sequencing combined with bioinformatic tools, more circRNAs catch scholars' attention. In addition to cancer tissue, circRNAs can be obtained from plasma, blood, urine, saliva, and other body fluids. Abnormal circulating circRNAs expression has been elucidated to be significantly connected with a variety of human cancer. Hence, it is worth investigating whether circulating circRNAs participate in the adjustment of the glucose metabolism of cancer.

\section{Cancer Stem Cells and Cancer Glucose Metabolism}

Previous studies have found that cell metabolism is closely related to the characteristics of "stemness". High levels of glucose uptake can interfere with the natural differentiation of cancer stem cells (CSC), laying the foundation for the Warburg effect. ${ }^{114}$ CircRNA is related to CSC, for example, circBIRC6 combines with miR-34 and miR-145 in human embryonic stem cells (hESCs) to maintain the pluripotency of stem cells and inhibit differentiation to induce the Warburg effect. ${ }^{69}$ Besides, circBIRC6 has multiple miRNA binding sites such as let-7, miR-92, and miR-103, and its biosynthesis is regulated by genes related to pluripotency, which can be used to further explore the inducing mechanism of Warburg.

\section{Exosomes and Cancer Glucose Metabolism}

In recent 5 years, many circRNAs have been reported to be enriched in exosomes, and the mystery of which has become a research hotspot. ${ }^{115}$ Exosomes can transport ncRNAs to tumor cells, induce mutational metabolism in 
cancer, promote growth and deliver essential nutrients. New studies have shown that when exosomes are upregulated in pancreatic cancer or prostate cancer cells, OXPHOS is inhibited and aerobic glycolysis is promoted. ${ }^{116}$ It is suggested that exosomes may regulate tumor cell metabolism through the transportation of circRNA and the regulation of signaling pathways, and the potential mechanism is expected to be further studied.

\section{Therapeutic Significance and Intervention of Cancer Cell Glucose Metabolism}

The circRNA in this article regulates tumor metabolism through various pathways and has clinical significance for targeted tumor therapy. Puerarin, sevoflurane, and propofol can inhibit BC, lung cancer, and glioma respectively, by inhibiting their targeted circRNA to interfere with tumor glucose metabolism and growth. More importantly, compared with other non-coding RNAs (such as lncRNA), circRNA is not easily degraded due to its closed structure. A small number of circRNAs can inhibit a large number of miRNAs. These studies provide new ideas for the exploration of highly effective anticancer drugs.

\section{Abbreviations}

APC, Antigen presenting cell; BC, bladder cancer; BxPC3, human orthotopic pancreatic adenocarcinoma cell 3; $\mathrm{CC}$, cervical cancer; CDK6, Cyclin-dependent Kinase 6; ceRNA, competing endogenous RNA; circRNA, circular RNA; CRC, colorectal cancer; ECAR, Extracellular acidification rate; EMT, Epithelial-mesenchymal transformation; ENO1, enolase 1; ESCC, esophageal squamous cell carcinoma; EWSR1, EWS RNA binding protein 1; FLOT2, Flotillin 2; G6PI, glucose-6-phosphate isomerase; GSK-3 $\beta$, glycogen synthase kinase-3 $\beta$; GLUT, glucose transporter; HCC, hepatocellular carcinoma; HIF1, hypoxia-inducing factor 1; HK, Hexokinase; HUVECs, human umbilical cord vascular endothelial cells; IGF-1, insulin-like growth factor 1; IL6R, interleukin-6 receptor; KLF2, krueppel-like factor 2; LDH, lactate dehydrogenase; LDHA, Lactate dehydrogenase A; LIF, Leukemia inhibitory Factor; lncRNA, Long non-coding RNA; LUAD, lung adenocarcinoma; MCT, Monocarboxylate transporter; NPC, nasopharyngeal carcinoma; ncRNAs, noncoding RNAs; NB, neuroblastoma; NSCLC, nonsmall cell lung cancer; OSCC, oral squamous cell carcinoma; OXPHOS, oxidative phosphorylation; PCa, prostatic cancer; PDK, Pyruvate dehydrogenase kinase;
PKM2, Pyruvate kinase M2; PGK, Phosphoglycerate kinase; PFK2, Phosphate Fructose Kinase 2; PI3K, Phosphatidylinositol 3 Kinase; PTC, papillary thyroid carcinoma; STAT3, signal transducer activator transcription 3; TAC, tricarboxylic acid; TFs, transcription factors; TGF- $\beta$, transforming growth factor- $\beta$; TNBC, triple negative breast cancer.

\section{Acknowledgments}

This work is supported by National Natural Science Foundation of China grants (Nos. 82073000 and 81972542), National Science Foundation of Sichuan Province (No. 2020JDRC0018 and 2020YFS0171), Clinical Project of West China College of Stomatology, Sichuan University (LCYJ2019-8), and Exploration and research projects of West China College of Stomatology, Sichuan University (LCYJ2020-YJ-1).

\section{Author Contributions}

All authors made a significant contribution to the work reported, whether that is in the conception, and interpretation; took part in drafting, revising, or critically reviewing the article; gave final approval of the version to be published; have agreed on the journal to which the article has been submitted; and agree to be accountable for all aspects of the work.

\section{Disclosure}

The authors report no conflicts of interest in this work.

\section{References}

1. Pamudurti NR, Bartok O, Jens M, et al. Translation of CircRNAs. Mol Cell. 2017;66(1):9-21.e27. doi:10.1016/j.molcel.2017.02.021

2. Kroemer G, Pouyssegur J. Tumor cell metabolism: cancer's achilles' heel. Cancer Cell. 2008;13(6):472-482. doi:10.1016/j.ccr.2008.05.005

3. DeBerardinis RJ, Thompson CB. Cellular metabolism and disease: what do metabolic outliers teach us? Cell. 2012;148(6):1132-1144. doi:10.1016/j.cell.2012.02.032

4. Koppenol WH, Bounds PL, Dang CV. Otto warburg's contributions to current concepts of cancer metabolism. Nat Rev Cancer. 2011;11 (5):325-337. doi:10.1038/nrc3038

5. Hsu PP, Sabatini DM. Cancer cell metabolism: warburg and beyond. Cell. 2008;134(5):703-707. doi:10.1016/j.cell.2008.08.021

6. Brooks GA. Cell-cell and intracellular lactate shuttles. $J$ Physiol. 2009;587(Pt 23):5591-5600. doi:10.1113/jphysiol.2009.178350

7. Bonuccelli G, Tsirigos A, Whitaker-Menezes D, et al. Ketones and lactate "fuel" tumor growth and metastasis: evidence that epithelial cancer cells use oxidative mitochondrial metabolism. Cell Cycle. 2010;9(17):3506-3514. doi:10.4161/cc.9.17.12731

8. Pfeiffer T, Schuster S, Bonhoeffer S. Cooperation and competition in the evolution of ATP-producing pathways. Science. 2001;292 (5516):504-507. doi:10.1126/science.1058079 
9. Jose C, Bellance N, Rossignol R. Choosing between glycolysis and oxidative phosphorylation: a tumor's dilemma? Biochim Biophys Acta. 2011;1807(6):552-561. doi:10.1016/j.bbabio.2010.10.012

10. Herst PM, Berridge MV. Cell surface oxygen consumption: a major contributor to cellular oxygen consumption in glycolytic cancer cell lines. Biochim Biophys Acta. 2007;1767(2):170-177. doi:10.1016/j.bbabio.2006.11.018

11. Griguer CE, Oliva CR, Gillespie GY. Glucose metabolism heterogeneity in human and mouse malignant glioma cell lines. $J$ Neurooncol. 2005;74(2):123-133. doi:10.1007/s11060-0046404-6

12. Zu XL, Guppy M. Cancer metabolism: facts, fantasy, and fiction. Biochem Biophys Res Commun. 2004;313(3):459-465. doi:10.1016/j.bbrc.2003.11.136

13. Moreno-Sánchez R, Rodríguez-Enríquez S, Marín-Hernández A, Saavedra E. Energy metabolism in tumor cells. FEBS J. 2007;274 (6):1393-1418. doi:10.1111/j.1742-4658.2007.05686.x

14. Hansen TB, Jensen TI, Clausen BH, et al. Natural RNA circles function as efficient microRNA sponges. Nature. 2013;495 (7441):384-388. doi:10.1038/nature11993

15. Shang Q, Yang Z, Jia R, Ge S. The novel roles of circRNAs in human cancer. Mol Cancer. 2019;18(1):6. doi:10.1186/s12943018-0934-6

16. Shi X, Wang B, Feng X, Xu Y, Lu K, Sun M. circRNAs and exosomes: a mysterious frontier for human cancer. Mol Ther Nucleic Acids. 2020;19:384-392. doi:10.1016/j.omtn.2019.11.023

17. Meng J, Chen S, Han JX, et al. Twist1 regulates vimentin through Cul2 circular RNA to promote EMT in hepatocellular carcinoma. Cancer Res. 2018;78(15):4150-4162. doi:10.1158/0008-5472. CAN-17-3009

18. Memczak S, Jens M, Elefsinioti A, et al. Circular RNAs are a large class of animal RNAs with regulatory potency. Nature. 2013;495(7441):333-338. doi:10.1038/nature11928

19. Mueckler M, Caruso C, Baldwin SA, et al. Sequence and structure of a human glucose transporter. Science. 1985;229 (4717):941-945. doi:10.1126/science.3839598

20. Hammond KD, Balinsky D. Isozyme studies of several enzymes of carbohydrate metabolism in human adult and fetal tissues, tumor tissues, and cell cultures. Cancer Res. 1978;38(5):1323.

21. Alpers JB, Wu R, Racker E. Regulatory mechanisms in carbohydrate metabolism. VI. Glycogen metabolism in HeLa cells. J Biol Chem. 1963;238:2274-2280. doi:10.1016/S0021-9258(19)679652

22. Vander Heiden MG. Targeting cancer metabolism: a therapeutic window opens. Nat Rev Drug Discov. 2011;10(9):671-684. doi:10.1038/nrd3504

23. Mortimer JE, Dehdashti F, Siegel BA, Katzenellenbogen JA, Fracasso P, Welch MJ. Positron emission tomography with 2-[18F]Fluoro-2-deoxy-D-glucose and 16alpha-[18F]fluoro17beta-estradiol in breast cancer: correlation with estrogen receptor status and response to systemic therapy. Clin Cancer Res. 1996;2(6):933-939.

24. Barron CC, Bilan PJ, Tsakiridis T, Tsiani E. Facilitative glucose transporters: implications for cancer detection, prognosis and treatment. Metabolism. 2016;65(2):124-139. doi:10.1016/j. metabol.2015.10.007

25. Carvalho KC, Cunha IW, Rocha RM, et al. GLUT1 expression in malignant tumors and its use as an immunodiagnostic marker. Clinics. 2011;66(6):965-972. doi:10.1590/S180759322011000600008

26. Ebstensen RD, Plagemann PG, Cytochalasin B. inhibition of glucose and glucosamine transport. Proc Natl Acad Sci U S A. 1972;69(6):1430-1434. doi:10.1073/pnas.69.6.1430

27. Stoll L, Sobel J, Rodriguez-Trejo A, et al. Circular RNAs as novel regulators of $\beta$-cell functions in normal and disease conditions. Mol Metab. 2018;9:69-83. doi:10.1016/j.molmet.2018.01.010
28. Zhao X, Lu C, Chu W, et al. MicroRNA-124 suppresses proliferation and glycolysis in non-small cell lung cancer cells by targeting AKT-GLUT1/HKII. Tumour Biol. 2017;39 (5):1010428317706215. doi:10.1177/1010428317706215

29. Zheng Q, Bao C, Guo W, et al. Circular RNA profiling reveals an abundant circHIPK 3 that regulates cell growth by sponging multiple miRNAs. Nat Commun. 2016;7:11215. doi:10.1038/ ncomms 11215

30. Zeng $\mathrm{Y}, \mathrm{Du} \mathrm{WW}, \mathrm{Wu} \mathrm{Y}$, et al. A circular RNA binds to and activates AKT phosphorylation and nuclear localization reducing apoptosis and enhancing cardiac repair. Theranostics. 2017;7 (16):3842-3855. doi:10.7150/thno. 19764

31. Jin M, Shi C, Yang C, Liu J, Huang G. Upregulated circRNA ARHGAP10 predicts an unfavorable prognosis in NSCLC through regulation of the miR-150-5p/GLUT-1 axis. Mol Ther Nucleic Acids. 2019;18:219-231. doi:10.1016/j.omtn.2019.08.016

32. Yuan G, Zhao Y, Wu D, Gao C. Mir-150 up-regulates Glut1 and increases glycolysis in osteosarcoma cells. Asian Pac J Cancer Prev. 2017;18(4):1127-1131. doi:10.22034/ APJCP.2017.18.4.1127

33. Ma J, Qi G, Li L, Novel Serum A. Exosomes-based biomarker hsa_circ_0002130 facilitates osimertinib-resistance in non-small cell lung cancer by sponging miR-498. Onco Targets Ther. 2020;13:5293-5307. doi:10.2147/OTT.S243214

34. Zhang ZJ, Zhang YH, Qin XJ, Wang YX, Fu J. CircularRNA circDENND4C facilitates proliferation, migration and glycolysis of colorectal cancer cells through miR-760/GLUT1 axis. Eur Rev Med Pharmacol Sci. 2020;24(5):2387-2400. doi:10.26355/ eurrev 202003 20506

35. Chen X, Yu J, Tian H, et al. Circle RNA hsa_circRNA_100290 serves as a ceRNA for miR-378a to regulate oral squamous cell carcinoma cells growth via Glucose transporter-1 (GLUT1) and glycolysis. $J$ Cell Physiol. 2019;234(11):19130-19140. doi: $10.1002 /$ jcp. 28692

36. Lin Q, Jiang H, Lin D. Circular RNA ITCH downregulates GLUT1 and suppresses glucose uptake in melanoma to inhibit cancer cell proliferation. $J$ Dermatolog Treat. 2019;32 (2):231-235.

37. Zhou J, Zhang S, Chen Z, He Z, Xu Y, Li Z. CircRNA-ENO1 promoted glycolysis and tumor progression in lung adenocarcinoma through upregulating its host gene ENO1. Cell Death Dis. 2019;10(12):885. doi:10.1038/s41419-019-2127-7

38. Dayton TL, Jacks T, Vander Heiden MG. PKM2, cancer metabolism, and the road ahead. EMBO Rep. 2016;17(12):1721-1730. doi:10.15252/embr.201643300

39. Luo W, Semenza GL. Emerging roles of PKM2 in cell metabolism and cancer progression. Trends Endocrinol Metab. 2012;23 (11):560-566. doi:10.1016/j.tem.2012.06.010

40. Chen X, Chen S, Yu D. Protein kinase function of pyruvate kinase M2 and cancer. Cancer Cell Int. 2020;20(1):523. doi:10.1186/ s12935-020-01612-1

41. Zahra K, Dey T, Ashish MSP, Pandey U. Pyruvate kinase M2 and cancer: the role of PKM2 in promoting tumorigenesis. Front Oncol. 2020;10:159. doi:10.3389/fonc.2020.00159

42. Iqbal MA, Gupta V, Gopinath P, Mazurek S, Bamezai RN. Pyruvate kinase M2 and cancer: an updated assessment. FEBS Lett. 2014;588(16):2685-2692. doi:10.1016/j.febslet.2014.04.011

43. Xiong Y, Lei QY, Zhao S, Guan KL. Regulation of glycolysis and gluconeogenesis by acetylation of PKM and PEPCK. Cold Spring Harb Symp Quant Biol. 2011;76:285-289. doi:10.1101/ sqb.2011.76.010942

44. Wang X, Zhang H, Yang H, et al. Exosome-delivered circRNA promotes glycolysis to induce chemoresistance through the miR-122-PKM2 axis in colorectal cancer. Mol Oncol. 2020;14 (3):539-555. doi:10.1002/1878-0261.12629 
45. Li Q, Pan X, Zhu D, Deng Z, Jiang R, Wang X. Circular RNA MAT2B promotes glycolysis and malignancy of hepatocellular carcinoma through the miR-338-3p/PKM2 axis under hypoxic stress. Hepatology. 2019;70(4):1298-1316. doi:10.1002/hep.30671

46. Fantin VR, St-Pierre J, Leder P. Attenuation of LDH-A expression uncovers a link between glycolysis, mitochondrial physiology, and tumor maintenance. Cancer Cell. 2006;9 (6):425-434. doi:10.1016/j.ccr.2006.04.023

47. Wei Y, Zhang Y, Meng Q, Cui L, Xu C. Hypoxia-induced circular RNA has_circRNA_403658 promotes bladder cancer cell growth through activation of LDHA. Am J Transl Res. 2019;11 (11):6838-6849.

48. Jin C, Dong D, Yang Z, Xia R, Tao S, Piao M. CircMYC regulates glycolysis and cell proliferation in melanoma. Cell Biochem Biophys. 2020;78(1):77-88. doi:10.1007/s12013-01900895-0

49. Elstrom RL, Bauer DE, Buzzai M, et al. Akt stimulates aerobic glycolysis in cancer cells. Cancer Res. 2004;64(11):3892-3899. doi:10.1158/0008-5472.CAN-03-2904

50. Altomare DA, Testa JR. Perturbations of the AKT signaling pathway in human cancer. Oncogene. 2005;24(50):7455-7464. doi:10.1038/sj.onc.1209085

51. Cho $\mathrm{H}, \mathrm{Mu} \mathrm{J}, \mathrm{Kim} \mathrm{JK}$, et al. Insulin resistance and a diabetes mellitus-like syndrome in mice lacking the protein kinase Akt2 (PKB beta). Science. 2001;292(5522):1728-1731. doi:10.1126/ science.292.5522.1728

52. Yang C, Sudderth J, Dang T, Bachoo RM, McDonald JG, DeBerardinis RJ. Glioblastoma cells require glutamate dehydrogenase to survive impairments of glucose metabolism or Akt signaling. Cancer Res. 2009;69(20):7986-7993. doi:10.1158/ 0008-5472.CAN-09-2266

53. Ou R, Mo L, Tang H, et al. circRNA-AKT1 sequesters miR-942$5 \mathrm{p}$ to upregulate AKT1 and promote cervical cancer progression. Mol Ther Nucleic Acids. 2020;20:308-322. doi:10.1016/j. omtn.2020.01.003

54. Bian A, Wang Y, Liu J, et al. Circular RNA Complement Factor $\mathrm{H}(\mathrm{CFH})$ promotes glioma progression by sponging miR-149 and regulating AKT1. Med Sci Monit. 2018;24:5704-5712. doi:10.12659/MSM.910180

55. Yang X, Liu L, Zou H, Zheng YW, Wang KP. circZFR promotes cell proliferation and migration by regulating miR-511/AKT1 axis in hepatocellular carcinoma. Dig Liver Dis. 2019;51 (10):1446-1455. doi:10.1016/j.dld.2019.04.012

56. Zhang L, Zhou Q, Qiu Q, et al. CircPLEKHM3 acts as a tumor suppressor through regulation of the miR-9/BRCA1/DNAJB6/ KLF4/AKT1 axis in ovarian cancer. Mol Cancer. 2019;18 (1):144. doi:10.1186/s12943-019-1080-5

57. Yang F, Zhang H, Mei Y, Wu M. Reciprocal regulation of HIF-1 $\alpha$ and lincRNA-p21 modulates the warburg effect. Mol Cell. 2014;53(1):88-100. doi:10.1016/j.molcel.2013.11.004

58. Stubbs M, Griffiths JR. The altered metabolism of tumors: HIF-1 and its role in the warburg effect. Adv Enzyme Regul. 2010;50 (1):44-55. doi:10.1016/j.advenzreg.2009.10.027

59. Kim J-W, Gao P, Liu Y-C, Semenza GL, Dang CV. Hypoxiainducible factor 1 and dysregulated c-Myc cooperatively induce vascular endothelial growth factor and metabolic switches hexokinase 2 and pyruvate dehydrogenase kinase 1. Mol Cell Biol. 2007;27(21):7381-7393. doi:10.1128/MCB.00440-07

60. Dang RY, Liu FL, Li Y. Circular RNA hsa_circ_0010729 regulates vascular endothelial cell proliferation and apoptosis by targeting the miR-186/HIF-1 $\alpha$ axis. Biochem Biophys Res Commun. 2017;490(2):104-110. doi:10.1016/j.bbrc.2017.05.164

61. Liang G, Liu Z, Tan L, Su AN, Jiang WG, Gong C. HIF1 $\alpha$ associated circDENND4C promotes proliferation of breast cancer cells in hypoxic environment. Anticancer Res. 2017;37 (8):4337-4343. doi:10.21873/anticanres.11827
62. Ren S, Liu J, Feng Y, et al. Knockdown of circDENND4C inhibits glycolysis, migration and invasion by up-regulating miR-200b/c in breast cancer under hypoxia. J Exp Clin Cancer Res. 2019;38(1):388. doi:10.1186/s13046-019-1398-2

63. Zhao Y, Zhong R, Deng C, Zhou Z. Circle RNA circABCB10 modulates PFN2 to promote breast cancer progression, as well as aggravate radioresistance through facilitating glycolytic metabolism via miR-223-3p. Cancer Biother Radiopharm. 2020. doi:10.1089/cbr.2019.3389

64. Shangguan H, Feng H, Lv D, Wang J, Tian T, Wang X. Circular RNA circSLC25A16 contributes to the glycolysis of non-smallcell lung cancer through epigenetic modification. Cell Death Dis. 2020;11(6):437. doi:10.1038/s41419-020-2635-5

65. Dang CV, Le A, Gao P. MYC-induced cancer cell energy metabolism and therapeutic opportunities. Clin Cancer Res. 2009;15(21):6479-6483. doi:10.1158/1078-0432.CCR-090889

66. Yeung SJ, Pan J, Lee MH. Roles of p53, MYC and HIF-1 in regulating glycolysis - the seventh hallmark of cancer. Cell Mol Life Sci. 2008;65(24):3981-3999. doi:10.1007/s00018-008-8224$\mathrm{x}$

67. Li Z, Zhang H. Reprogramming of glucose, fatty acid and amino acid metabolism for cancer progression. Cell Mol Life Sci. 2016;73(2):377-392. doi:10.1007/s00018-015-2070-4

68. Xie H, Ren X, Xin S, et al. Emerging roles of circRNA_001569 targeting miR-145 in the proliferation and invasion of colorectal cancer. Oncotarget. 2016;7(18):26680-26691. doi:10.18632/ oncotarget.8589

69. Yu C-Y, Li T-C, Wu Y-Y, et al. The circular RNA circBIRC6 participates in the molecular circuitry controlling human pluripotency. Nat Commun. 2017;8(1):1149. doi:10.1038/ s41467-017-01216-w

70. Li H, Yang F, Hu A, et al. Therapeutic targeting of circ-CUX1/ EWSR1/MAZ axis inhibits glycolysis and neuroblastoma progression. EMBO Mol Med. 2019;11(12):e10835-e10835. doi:10.15252/emmm.201910835

71. Zheng $\mathrm{X}$, Huang $\mathrm{M}$, Xing $\mathrm{L}$, et al. The circRNA circSEPT9 mediated by E2F1 and EIF4A3 facilitates the carcinogenesis and development of triple-negative breast cancer. Mol Cancer. 2020;19(1):73. doi:10.1186/s12943-020-01183-9

72. Yang Q, Du WW, Wu N, et al. A circular RNA promotes tumorigenesis by inducing c-myc nuclear translocation. Cell Death Differ. 2017;24(9):1609-1620. doi:10.1038/cdd.2017.86

73. Yang Y, Gao X, Zhang M, et al. Novel role of FBXW7 circular RNA in repressing glioma tumorigenesis. J Natl Cancer Inst. 2018;110(3):304-315. doi:10.1093/jnci/djx166

74. Yang J, Ahmed A, Poon E, et al. Small-molecule activation of p53 blocks hypoxia-inducible factor 1alpha and vascular endothelial growth factor expression in vivo and leads to tumor cell apoptosis in normoxia and hypoxia. Mol Cell Biol. 2009;29 (8):2243-2253. doi:10.1128/MCB.00959-08

75. Zhou S, Kachhap S, Singh KK. Mitochondrial impairment in p53-deficient human cancer cells. Mutagenesis. 2003;18 (3):287-292. doi:10.1093/mutage/18.3.287

76. Ma W, Sung HJ, Park JY, Matoba S, Hwang PM. A pivotal role for p53: balancing aerobic respiration and glycolysis. J Bioenerg Biomembr. 2007;39(3):243-246. doi:10.1007/s10863-007-9083-0

77. Li H, Jin X, Liu B, Zhang P, Chen W, Li Q. CircRNA CBL.11 suppresses cell proliferation by sponging miR-6778-5p in colorectal cancer. BMC Cancer. 2019;19(1):826. doi:10.1186/s12885019-6017-2

78. Tian S, Han G, Lu L, Meng X. Circ-FOXM1 contributes to cell proliferation, invasion, and glycolysis and represses apoptosis in melanoma by regulating miR-143-3p/FLOT2 axis. World J Surg Oncol. 2020;18(1):56. doi:10.1186/s12957-020-01832-9 
79. Kawada K, Toda K, Sakai Y. Targeting metabolic reprogramming in KRAS-driven cancers. Int J Clin Oncol. 2017;22(4):651-659. doi:10.1007/s10147-017-1156-4

80. Huang M, Zhong Z, Lv M, Shu J, Tian Q, Chen J. Comprehensive analysis of differentially expressed profiles of lncRNAs and circRNAs with associated co-expression and ceRNA networks in bladder carcinoma. Oncotarget. 2016;7(30):47186-47200. doi:10.18632/oncotarget.9706

81. Li H, Li J, Jia S, et al. miR675 upregulates long noncoding RNA H19 through activating EGR1 in human liver cancer. Oncotarget. 2015;6(31):31958-31984. doi:10.18632/oncotarget.5579

82. Zhong Z, Huang M, Lv M, et al. Circular RNA MYLK as a competing endogenous RNA promotes bladder cancer progression through modulating VEGFA/VEGFR2 signaling pathway. Cancer Lett. 2017;403:305-317. doi:10.1016/j. canlet.2017.06.027

83. Jiang S, Zhang L-F, Zhang H-W, et al. A novel miR-155/miR-143 cascade controls glycolysis by regulating hexokinase 2 in breast cancer cells. EMBO J. 2012;31(8):1985-1998. doi:10.1038/ emboj.2012.45

84. Yang ZG, Awan FM, Du WW, et al. The circular RNA interacts with STAT3, increasing its nuclear translocation and wound repair by modulating Dnmt3a and miR-17 function. Mol Ther. 2017;25 (9):2062-2074. doi:10.1016/j.ymthe.2017.05.022

85. Liu X, Zhang A, Xiang J, Lv Y, Zhang X. miR-451 acts as a suppressor of angiogenesis in hepatocellular carcinoma by targeting the IL-6R-STAT3 pathway. Oncol Rep. 2016;36 (3):1385-1392. doi:10.3892/or.2016.4971

86. Deng N, Li L, Gao J, et al. Hsa_circ_0009910 promotes carcinogenesis by promoting the expression of miR-449a target IL6R in osteosarcoma. Biochem Biophys Res Commun. 2018;495 (1):189-196. doi:10.1016/j.bbrc.2017.11.028

87. Chen G, Shi Y, Zhang Y, Sun J. CircRNA_100782 regulates pancreatic carcinoma proliferation through the IL6-STAT3 pathway. Onco Targets Ther. 2017;10:5783-5794. doi:10.2147/ OTT.S150678

88. Robey RB, Hay N. Is Akt the "warburg kinase"?-Akt-energy metabolism interactions and oncogenesis. Semin Cancer Biol. 2009;19(1):25-31. doi:10.1016/j.semcancer.2008.11.010

89. Yang X, Cheng Y, Li P, et al. A lentiviral sponge for miRNA-21 diminishes aerobic glycolysis in bladder cancer T24 cells via the PTEN/PI3K/AKT/mTOR axis. Tumor Biol. 2015;36(1):383-391. doi:10.1007/s13277-014-2617-2

90. Robitaille AM, Christen S, Shimobayashi M, et al. Quantitative phosphoproteomics reveal mTORC1 activates de novo pyrimidine synthesis. Science. 2013;339(6125):1320-1323. doi:10.1126/ science. 1228771

91. Zhang X, Wang S, Wang H, et al. Circular RNA circNRIP1 acts as a microRNA-149-5p sponge to promote gastric cancer progression via the AKT1/mTOR pathway. Mol Cancer. 2019;18(1):20. doi:10.1186/s12943-018-0935-5

92. Wang H, Yan X, Zhang H, Zhan X. CircRNA circ_0067934 overexpression correlates with poor prognosis and promotes thyroid carcinoma progression. Med Sci Monit. 2019;25:1342-1349. doi:10.12659/MSM.913463

93. Chen L, Zhang S, Wu J, et al. circRNA_100290 plays a role in oral cancer by functioning as a sponge of the miR-29 family. Oncogene. 2017;36(32):4551-4561. doi:10.1038/onc.2017.89

94. Liu W, Zhang J, Zou C, et al. Microarray expression profile and functional analysis of circular RNAs in osteosarcoma. Cell Physiol Biochem. 2017;43(3):969-985. doi:10.1159/000481650

95. Gao Y, Ma H, Gao Y, et al. CircRNA Circ_0001721 promotes the progression of osteosarcoma through miR-372-3p/MAPK7 axis. Cancer Manag Res. 2020;12:8287-8302. doi:10.2147/CMAR. S244527
96. Qiu Y, Pu C, Li Y, Qi B. Construction of a circRNA-miRNAmRNA network based on competitive endogenous RNA reveals the function of circRNAs in osteosarcoma. Cancer Cell Int. 2020;20:48. doi:10.1186/s12935-020-1134-1

97. Sun J, Yuan X, Li X, et al. Comparative transcriptome analysis of the global circular RNAs expression profiles between SHEE and SHEEC cell lines. Am J Transl Res. 2017;9(11):5169-5179.

98. Pate KT, Stringari C, Sprowl-Tanio S, et al. Wnt signaling directs a metabolic program of glycolysis and angiogenesis in colon cancer. EMBO J. 2014;33(13):1454-1473. doi:10.15252/ embj. 201488598

99. Rennoll S, Yochum G. Regulation of MYC gene expression by aberrant Wnt/ $\beta$-catenin signaling in colorectal cancer. World J Biol Chem. 2015;6(4):290-300. doi:10.4331/wjbc.v6.i4.290

100. Bi W, Huang J, Nie C, et al. CircRNA circRNA 102171 promotes papillary thyroid cancer progression through modulating CTNNBIP1-dependent activation of $\beta$-catenin pathway. $J$ Exp Clin Cancer Res. 2018;37(1):275. doi:10.1186/s13046-0180936-7

101. Wang M, Chen B, Ru Z, Cong L. CircRNA circ-ITCH suppresses papillary thyroid cancer progression through $\mathrm{miR}-22-3 \mathrm{p} / \mathrm{CBL} / \beta$ catenin pathway. Biochem Biophys Res Commun. 2018;504 (1):283-288. doi:10.1016/j.bbrc.2018.08.175

102. Zhu Q, Lu G, Luo Z, et al. CircRNA circ_0067934 promotes tumor growth and metastasis in hepatocellular carcinoma through regulation of $\mathrm{miR}-1324 / \mathrm{FZD} 5 / \mathrm{Wnt} / \beta$-catenin axis. Biochem Biophys Res Commun. 2018;497(2):626-632. doi:10.1016/j. bbrc.2018.02.119

103. Yao Y, Hua Q, Zhou Y. CircRNA has_circ_0006427 suppresses the progression of lung adenocarcinoma by regulating miR-6783$3 \mathrm{p} / \mathrm{DKK} 1$ axis and inactivating Wnt/ $\beta$-catenin signaling pathway. Biochem Biophys Res Commun. 2019;508(1):37-45. doi:10.1016/ j.bbrc.2018.11.079

104. Li Y, Zhang J, Pan S, Zhou J, Diao X, Liu S. CircRNA CDR1as knockdown inhibits progression of non-small-cell lung cancer by regulating miR-219a-5p/SOX5 axis. Thorac Cancer. 2020;11 (3):537-548. doi:10.1111/1759-7714.13274

105. Zhong Q, Huang J, Wei J, Wu R. Circular RNA CDR1as sponges miR-7-5p to enhance E2F3 stability and promote the growth of nasopharyngeal carcinoma. Cancer Cell Int. 2019;19:252. doi:10.1186/s12935-019-0959-y

106. Yao Z, Luo J, Hu K, et al. ZKSCAN1 gene and its related circular RNA (circZKSCAN1) both inhibit hepatocellular carcinoma cell growth, migration, and invasion but through different signaling pathways. Mol Oncol. 2017;11(4):422-437. doi:10.1002/18780261.12045

107. Liang Y, Wang H, Chen B, et al. circDCUN1D4 suppresses tumor metastasis and glycolysis in lung adenocarcinoma by stabilizing TXNIP expression. Mol Ther Nucleic Acids. 2021;23:355-368. doi:10.1016/j.omtn.2020.11.012

108. Pan Z, Cai J, Lin J, et al. A novel protein encoded by circFNDC3B inhibits tumor progression and EMT through regulating snail in colon cancer. Mol Cancer. 2020;19(1):71. doi:10.1186/s12943-020-01179-5

109. Rong D, Sun H, Li Z, et al. An emerging function of circRNA-miRNAs-mRNA axis in human diseases. Oncotarget. 2017;8(42):73271-73281. doi:10.18632/oncotarget.19154

110. Du L, Zhang L, Sun F. Puerarin inhibits the progression of bladder cancer by regulating circ_0020394/miR-328-3p/NRBP1 axis. Cancer Biother Radiopharm. 2020. doi:10.1089/ cbr.2019.3382

111. Zhao H, Wei H, He J, et al. Propofol disrupts cell carcinogenesis and aerobic glycolysis by regulating circTADA2A/miR-455-3p/ FOXM1 axis in lung cancer. Cell Cycle. 2020;19(19):2538-2552. doi:10.1080/15384101.2020.1810393 
112. Li H, Xia T, Guan Y, Yu Y. Sevoflurane regulates glioma progression by Circ_0002755/miR-628-5p/MAGT1 axis. Cancer Manag Res. 2020;12:5085-5098. doi:10.2147/CMAR.S242135

113. Du J, Zhang L, Ma H, Wang Y, Wang P. Lidocaine suppresses cell proliferation and aerobic glycolysis by regulating circHOMER1/ miR-138-5p/HEY1 axis in colorectal cancer. Cancer Manag Res. 2020;12:5009-5022. doi:10.2147/CMAR.S244973

114. Riester M, Xu Q, Moreira A, Zheng J, Michor F, Downey RJ. The warburg effect: persistence of stem-cell metabolism in cancers as a failure of differentiation. Ann Oncol. 2018;29(1):264-270. doi:10.1093/annonc/mdx645
115. Lener T, Gimona M, Aigner L, et al. Applying extracellular vesicles based therapeutics in clinical trials - an ISEV position paper. J Extracell Vesicles. 2015;4:30087. doi:10.3402/jev. v4.30087

116. Zhao H, Yang L, Baddour J, et al. Tumor microenvironment derived exosomes pleiotropically modulate cancer cell metabolism. eLife. 2016;5:e10250. doi:10.7554/eLife.10250

\section{Publish your work in this journal}

OncoTargets and Therapy is an international, peer-reviewed, open access journal focusing on the pathological basis of all cancers, potential targets for therapy and treatment protocols employed to improve the management of cancer patients. The journal also focuses on the impact of management programs and new therapeutic

Submit your manuscript here: https://www.dovepress.com/oncotargets-and-therapy-journal agents and protocols on patient perspectives such as quality of life, adherence and satisfaction. The manuscript management system is completely online and includes a very quick and fair peer-review system, which is all easy to use. Visit http://www.dovepress.com/ testimonials.php to read real quotes from published authors. 